\title{
Two or four injections of leukocyte-poor platelet-rich plasma for osteoarthritic knee did not affect changes of synovial cytokines and growth factors but similarly improved clinical outcomes
}

Srihatach Ngarmukos

Chulalongkorn University

Chotetawan Tanavalee

King Chulalongkorn Memorial Hospital

Chavarin Amarase

King Chulalongkorn Memorial Hospital

Suphattra Phakham

Chulalongkorn University

Warayapa Mingsiritham

Chulalongkorn University

Rangsima Reantragoon

Chulalongkorn University

Nitigorn Leearamwat

Chulalongkorn University

Thidarat Kongkaew

Chulalongkorn University

Kittipan Tharakhet

Chulalongkorn University

Sittisak Honsawek

Chulalongkorn University

Sinsuda Dechsupa

Chulalongkorn University

Aree Tanavalee ( $\boldsymbol{D}$ areetana@hotmail.com )

Chulalongkorn University

\section{Research Article}

Keywords: Platelet-rich plasma, PRP, knee, osteoarthritis, OA, treatment, cytokine, growth factor

Posted Date: August 3rd, 2021 
DOl: https://doi.org/10.21203/rs.3.rs-745615/v1

License: (c) (1) This work is licensed under a Creative Commons Attribution 4.0 International License. Read Full License 


\section{Abstract}

We compared two and four intra-articular injections of platelet-rich plasma (PRP) in terms of changes of synovial cytokines and clinical outcomes. One hundred twenty-five patients having knee OA underwent leukocyte-poor PRP with a 6-week interval. Before each PRP injection, synovial fluid aspiration was collected for investigation. Patients were divided into two or four intra-articular PRP injections (group A and $B$, respectively). Changes in synovial cytokines and growth factors were compared with the baseline levels of both groups, and clinical outcomes were evaluated until one year. Ninety-four patients who had completed synovial fluid collection were included for final evaluation, 51 in group A and 43 in group B. There were no differences in mean age, gender, $\mathrm{BMI}$, and OA grading. The average platelet count in PRP was $430,000 / \mu \mathrm{L}$. There were no changes of synovial inflammatory cytokines (IL-1B, IL-6, IA-17A, and TNFalpha), anti-inflammatory cytokines (IL-4, IL-10, IL-13, and IL-1RA), and growth factors (TGF-B1, VEGF, PDGF-AA, and PDGF-BB) between baseline levels and six weeks in group A, and 18 weeks in group $B$. Both groups had significantly improved clinical outcomes from six weeks including visual analog scale (VAS), patient-reported outcome measure (PROM; WOMAC and SF-12), with a significant delayed improvement of performance-based measure [PBM; time up and go (TUG), 5-time sit to stand test (5×SST), and 3-minute walk test (3-min WT)]. In conclusion, two- or four-PRP injection at a 6-week interval for knee OA demonstrated no changes of synovial cytokines and growth factors but similarly improved clinical outcomes from 6 weeks until one year.

\section{Introduction}

Knee osteoarthritis $(\mathrm{OA})$ is a significant degenerative joint disease affecting patients' quality of life and daily functions ${ }^{1}$. Treatment options are based on the stage of the disease, the patient's condition, and available medical facilities. Therefore, recommended methods range from non-surgical to surgical treatments ${ }^{2-6}$. However, in mild to moderate knee OA, non-surgical treatment options are in good agreement between physicians and patients with a wide diversity of results reported in the literature ${ }^{2-4,6}$.

Although most significant guidelines do not include or recommend against platelet-rich plasma (PRP), a biologic product preparing from autologous blood ${ }^{5-7}$, PRP with a variation of preparation protocol, has become an attractive option for the non-surgical treatment of knee $\mathrm{OA}^{8-12}$. Studies regarding PRP applied in knee OA showed that it improved patient's clinical outcomes by releasing several growth factors and cytokines, promoting reparation and minimization of the inflammation occurring in the process of degenerative arthritis ${ }^{10-17}$. In addition, some studies reported that PRP injection for knee OA provided better pain relief and improved functional outcomes than placebo control, hyaluronic acid, or steroid injection ${ }^{18-20}$.

Due to the heterogenicity of PRP preparation and protocol in knee OA treatment, including the amount of collected blood, the method of centrifugation, the amount of white blood cell, the activation of PRP, the number of injection, and the interval of each injection, a current consensus of French-spoken physicians 
suggested that the property of PRP for knee OA treatment should be an activated, leukocyte-poor, and 4$8 \mathrm{~mL}$ in volume, and multiple PRP injections provide better results ${ }^{21}$.

The present study evaluated the changes of inflammatory, anti-inflammatory cytokines, and growth factors in the synovial fluid after two or four injections of activated, leukocyte-poor PRP at 6-week interval in knee OA patients, as well as patient's clinical outcomes, including pain relief using the visual analog scale (VAS), patient-reported outcome measure (PROM), and performance-based measure (PBM).

\section{Methods}

This study was approved by The Institutional Reviewed Board of The Faculty of Medicine, Chulalongkorn University, Bangkok, Thailand (COA No. 804/2020 and IRB No. 040/63). All methods and experimental protocols were carried out in accordance with relevant guidelines and regulations. Informed consent was obtained from all patients participated in the study. From January to May 2020, all out-patients who had knee OA and came to the orthopedic clinic were introduced to a biologic treatment of knee OA with intraarticular injection of PRP. They also were informed that the method and protocol of treatment was not a standard treatment for knee OA in this country. Selection criteria included 45-85 years of age, grade 1 to 4 of primary knee OA according to the Kellgren-Lawrence system ${ }^{22}$, > 90-degree arc of motion, $<10$ degree anatomical deformity in the coronal plane, and no skin lesion around the affected knee. In addition, all recruited patients had a 2-week washing-out period for any pain killers or no non-steroidal anti-inflammatory drugs (NSAIDs), as well as joint supplements. Also, patients agreed not to take these medications during FU. One hundred twenty-five patients were voluntary in this study.

The synovial fluid aspiration without an anesthetic agent was somewhat painful in the pilot study, especially in patients whose knees were not swollen. Therefore, patients were voluntary to choose 2injection protocol (group A) or 4-injection protocol (group B). Patients were evaluated for clinical parameters at week 0 before the PRP injection and serial follow-up (FU) until one year.

\section{PRP protocol and procedure}

A leukocyte-poor PRP was prepared by drawing a 30-mL of peripheral blood from the cubital vein. A 3-mL acid citrate dextrose (ACD) solution was added as an anticoagulant. The first centrifugation was performed at $100 \mathrm{G}$ for $10 \mathrm{~min}$ to collect the platelet-poor plasma (PPP). After collecting the PPP, the second centrifugation was again performed, setting at 400G for 8 min to collect the leukocyte-poor PRP, and a $0.1 \%$ volume of $\mathrm{CaCl}_{2}$ was added before the injection.

Under a sterile preparation, the intra-articular injection was performed via the superolateral direction. Before every injection of PRP at all FU visits, synovial fluid aspiration for molecular analysis was performed using a 23-gauge needle and a 5-mL syringe without anesthetic infiltration. First, a 1- to 2-mL synovial fluid was aspirated. The aspirated syringe was then dislodged from the hand, and then the syringe containing PRP was applied, and a PRP injection procedure was performed. All steps of 
aspiration and PRP injection were the same in all patients and all FU visits. Patients whose knee aspirations resulted in negative joint fluid or blood contamination were excluded from the studied group. The aspirated synovial fluid before the first PRP injection (week 0) was defined as the baseline. In contrast, the rest were described as parameters after each intervention according to the time of FU.

After the PRP injection, patients were allowed to maintain their daily activities as usual. No pain killers or anti-inflammatory drugs (NSAIDs) were permitted for any reason; otherwise, patients would be dropped out from the study. Patients in both groups were appointed for the next injection six weeks after the previous injection. Therefore, the PRP injection was performed at weeks 0 and 6 in group A and week 0 , week 6 , week 12, and week 18 in group B.

\section{Molecular study}

The synovial fluid sample was centrifuged at $3500 \mathrm{rpm}$ for $10 \mathrm{~min}$ and then stored at $-80^{\circ} \mathrm{C}$ until all the synovial fluid samples of all patients at all FUs were collected. Analysis for the level of cytokines was performed using the cytokine proteome array (Bio-Plex suspension array system, BioRad, Hercules, CA, USA), which was reported in $\mathrm{pg} / \mathrm{mL}$. In addition, the molecular study, including levels of synovial inflammatory cytokines (IL-1ß, IL-6, IA-17A, and TNF-alpha), anti-inflammatory cytokines (IL-4, IL-10, IL-13, and IL-1RA), and growth factors (TGF-B1, VEGF, PDGF-AA, and PDGF-BB) of each patient were evaluated. Molecular study of synovial fluid of patients in group A was compared between baseline values (week 0 before the first PRP injection) and those of week six before the second PRP injection. Similarly, the baseline levels of molecular study of patients in group B were compared to week six, week 12, and week 18 , before the second, the third, and the fourth PRP injections.

\section{Clinical outcomes}

The clinical outcomes were evaluated for clinical outcomes and followed until one year. The patient's subjective pain using a visual analog scale (VAS), patient's report outcome measure (PROM) using Western Ontario and McMaster Universities Osteoarthritis (WOMAC) index and Short Form-12 (SF-12), and performance-based measure (PBM) using time up and go (TUG), 5 -time sit to stand test (5×SST), and 3-minute walk test (3-min WT) were investigated before the first PRP injection (week 0 ) as baseline parameters. At six weeks, 12 weeks, 18 weeks, six months, and one year from the intervention, all parameters were evaluated and compared to baseline levels.

\section{Statistical analysis}

The GraphPad Prism version 9.0.2, GraphPad Software, San Diego, CA, USA, www.graphpad.com, was used for statistical analyses. Descriptive statistics were performed for patient's demographic data. The one-way ANOVA followed by Dunnett's multiple comparisons test was performed to evaluate parameters among each group's baseline and other FU visits. The two-way ANOVA followed by Šidák's multiple comparisons test was performed to assess differences between groups A ( 2 injections) and B (4 injections). 


\section{Results}

Among 125 patients in this studied group, 19 from 70 patients in group A and 12 from 55 patients in group $B$ were excluded due to the inability to collect synovial fluid before PRP injection at any time or blood contamination in synovial fluid. Therefore, 51 patients in group A and 43 patients in group $B$ were available for the evaluation. The patients' demographic data and radiographic grading of knee OA according to Kellgren-Lawrence $(K L)$, ranging from $K L 1, K L 2, K L 3$, and $K L 4$, were shown in Table 1 . Female patients were dominant, with an average age of 67 years. There were no differences between groups A and B in terms of mean age, gender, and BMI. The average PRP volume before injection was 6.7 $\mathrm{ml}$. The average platelet count in PRP was $430,000 / \mu \mathrm{L}$ which was equal to 3.8 times of whole blood level, and the average white and red blood cell counts were $200 / \mu \mathrm{L}, 30,000 / \mu \mathrm{L}$, respectively.

Regarding the molecular study of synovial fluid in both groups, all investigated parameters in group $\mathrm{A}$ were compared between week 0 and week six, which were defined as parameters after one PRP injection. All investigated parameters in group B were evaluated from baseline (week 0), week 6, week 12, and week 18, defined as parameters after one PRP injection, two PRP injections, and three PRP injections. There were no significant changes in inflammatory cytokines (IL-1ß, IL-6, IA-17A, and TNF-alpha), antiinflammatory cytokines (IL-4, IL-10, IL-13, and IL-1RA), and growth factors (TGF-B1, VEGF, PDGF-AA, and PDGF-BB) in group $A$ at six weeks compared to those at baseline as shown in Fig. 1. Similarly, there were no changes in the same investigated cytokines and growth factors at six weeks, 12 weeks, and 18 weeks compared to those at baseline, as shown in Fig. 2.

Both groups A and B had a similar pattern of improved clinical outcomes. The VAS and the PROM, including the WOMAC index and SF-12, significantly improved at six weeks after the baseline evaluation. However, the PBM, including TUG, and 5×SST showed significant improvement from 12 weeks, while the 3-min WT showed the latest progress from 18 weeks (Fig. 3). At one year FU, all parameters of clinical outcomes remained significantly improved from the baseline evaluation. Comparing clinical results between both groups A and B, all investigated parameters had similar significant improvement (Fig. 4).

\section{Discussion}

The present study was designed to evaluate changes in inflammatory cytokines (IL-1ß, IL-6, IA-17A, and TNF-alpha), anti-inflammatory cytokines (IL-4, IL-10, IL-13, and IL-1RA), and growth factors (TGF-B1, VEGF, PDGF-AA, and PDGF-BB) in synovial fluid and clinical outcomes related to two different protocols of leukocyte-poor PRP injection at a 6-week interval. Patients in group A (2-injection protocol) were compared to group B (4-injection protocol) and were followed until one year. The results demonstrated three aspects. Firstly, no changes in synovial inflammatory cytokines, anti-inflammatory cytokines, and growth factors in group A implied no changes of investigated parameters at six weeks after one PRP injection. Secondly, no changes in synovial inflammatory cytokines, anti-inflammatory cytokines, and growth factors in group B implied no changes of investigated parameters after one, two, and three consecutive PRP injections. Lastly, there were early significantly improved clinical outcomes from 6 
weeks, especially VAS and PROM (WOMAC index and SF-12), and delayed improved PBM (TUG, 5×SST, and 3-min WT) from 12 weeks, with no differences between both groups at all visits of FU until one year.

Studies have shown that the level of inflammatory cytokines in synovial fluid is related to the severity of osteoarthritis ${ }^{23-26}$. Thus, clinical symptoms of OA should relate directly to the level of these cytokines. The present study evaluated four synovial inflammatory cytokines, including IL-1B, IL-6, IA-17A, and TNFalpha, four synovial anti-inflammatory cytokines, including IL-4, IL-10, IL-13, and IL-1RA, and four synovial growth factors, including TGF-B1, VEGF, PDGF-AA, and PDGF-BB. Therefore, any changes of the molecular study from the baseline level related to multiple PRP injections of two different protocols would objectively reflect the effectiveness of PRP treatment. However, groups A and B had no significant changes in synovial cytokines and growth factors at all FU visits. In contrast, all patients have significantly improved clinical outcomes from 6 weeks until one year. So, we found no relationship between levels of synovial cytokines and clinical outcomes, as well as the number of PRP injections. Although the method of molecular research in the present study was a standard method in the biochemistry and immunology laboratories, the changed level cytokines and growth factors in synovial fluid might be too limited to be detected. Therefore, a molecular study using other joint tissues, such as the synovial membrane, may be more appropriate.

In the present study, similarly improved VAS, WOMAC index, and SF-12 after PRP treatment improvement agreed with several previous studies which reported significant pain relief and improved WOMAC index at one year of FU 8,9,20,27-29. A recent meta-analysis including 34 randomized controlled trials in 1,403 knees concluded that PRP provided better WOMAC than placebo and hyaluronic acid injection and better VAS than corticosteroid injection ${ }^{30}$. Besides the VAS and PROM, the PBM including TUG, 5×SST, and 3-min WT were added in the present study to evaluate objective parameters. These PBM reflected a patient's actual function without any bias related to the patient's attitude on PRP treatment. Although these three tests significantly improved, it took longer than the VAS and PROM to gain a significant value. The 3-min WT was found delayed significant change comparing to TUG and $5 \times$ SST. The mean time to do TUG and $5 \times$ SST in each FU visit took a much shorter time and less energy than 3-min WT. Therefore, more time and energy-consuming in the 3-min WT could be the primary cause to explain its delayed change among PBM. According to differences of substantial time of improvement among investigated parameters, it is the straight foreword that a patient should have significantly improved pain and PROM before gaining improved PBM, which relates directly to the consuming time, including TUG, and 3-min WT, respectively.

According to the PRP preparation protocol of the present study, we drew $30-\mathrm{mL}$ peripheral blood with double centrifugation, which resulted in a mean of $6.7 \mathrm{~mL}$ of PRP. It contained a 3.8-time higher platelet cell count with poor leukocyte count. This PRP property was partially similar to that recommended in the current consensus statement of PRP treatment of knee OA from French-speaking experts ${ }^{21}$. This consensus suggested that the PRP should be prepared with double centrifugation, resulting in $4-8 \mathrm{~mL}$, low leukocyte count, and 1- to 3-injection protocol several weeks apart. There were two different points of our study from the recommendation of the consensus. Firstly, the PRP in the present study has a lower platelet count than the recommended at 5-time or more concentration. Secondly, the present study 
included severe OA (KL 4) in both groups, which was not recommended by this consensus. However, in the severe OA subgroup, our selection criteria included only limited angular deformity resulting in minimal

symptoms of mechanical knee pain causing patients to undergo surgery ${ }^{31}$. The present study resulted in a favorable clinical outcome, regardless of lower platelet concentration in PRP, and no change of molecular study. We believe that several unclear factors affect the positive effects of this biological treatment, and further studies to analyze the conflict result between subjective and objective findings may be helpful.

The weakness of the present study includes no control group. This study was previously designed to prospectively evaluate changes of synovial cytokines and growth factors, as well as clinical outcomes after PRP injection in knee OA patients compared with the control group using intra-articular saline injection. However, the protocol which performs repetitive aspirations of synovial fluid and intra-articular injections of saline solution to the knee joint did not get approval from the IRB. Therefore, we had to modify the protocol, which compared baseline parameters with those evaluated at all visits of FU. Nevertheless, there are some strengths of the present study. Firstly, we evaluated synovial inflammatory cytokines, anti-inflammatory cytokines, and growth factors after single and three PRP injections which was an invasive, and technical demanding at the time of synovial fluid aspiration, resulting in indifference. Secondly, we demonstrated positive results of PRP with two different multiple injection protocols which few studies focused in this issue.

\section{Conclusions}

Two- or four-intra-articular injection protocol of leukocyte-poor PRP for treatment of knee OA demonstrated similarly no changes in synovial inflammatory cytokines, anti-inflammatory cytokines, and growth factors at six weeks, 18 weeks after the first PRP injection, respectively. However, significantly improved VAS, PROM (WOMAC and SF-12) from 6 weeks until one year of FU with delayed significantly improved PBM (TUG, 5×SST, and 3-min WT).

\section{Declarations}

\section{-Ethical Approval}

- This study was performed in line with the principles of the Declaration of Helsinki. Approval was granted by the Ethics Committee of Chulalongkorn University No IRB040/63.

\section{-Consent to Participate}

- Informed consent was obtained from all individual participants included in the study.

\section{-Consent to Publish}

- Patients signed informed consent regarding publishing their data. 


\section{-Authors Contributions}

- AT, SN, RR, and SH established the design and implemented the research and analysis of the results.

- SP processed IRB documents.

- AT, CT and CA performed knee aspiration and PRP injection.

- WM and SD collected VAS, PROM (WOMAC index, SF-12), and performed PBM (TUG, 5×SST, and 3$\min \mathrm{WT}$ ).

- SP, NL, TK, and KT performed PRP preparation and analysis.

- RR and KT performed a molecular study.

- $\mathrm{AT}, \mathrm{SN}, \mathrm{CT}, \mathrm{CA}, \mathrm{RR}$, and SH wrote the manuscript together.

\section{-Funding}

- This study has no funding support.

\section{-Competing Interests}

- All authors declare no conflict of interest in this study.

\section{-Availability of data and materials}

- The datasets generated in the current study are not publicly available because the PRP injection for knee osteoarthritis is not approved as a standard treatment in this country but are available from the corresponding author.

\section{References}

1 Hunter, D. J. \& Bierma-Zeinstra, S. Osteoarthritis. Lancet 393, 1745-1759, doi:10.1016/S01406736(19)30417-9 (2019).

2 Bannuru, R. R. et al. OARSI guidelines for the non-surgical management of knee, hip, and polyarticular osteoarthritis. Osteoarthritis Cartilage 27, 1578-1589, doi:10.1016/j.joca.2019.06.011 (2019).

3 Kan, H. S. et al. Non-surgical treatment of knee osteoarthritis. Hong Kong Med J 25, 127-133, doi:10.12809/hkmj187600 (2019).

4 Sprouse, R. A., Harris, G. D. \& Sprouse, G. D. E. A practical approach to knee OA. J Fam Pract 69, 327-334 (2020).

$5 \quad$ Kolasinski, S. L. et al. 2019 American College of Rheumatology/Arthritis Foundation Guideline for the Management of Osteoarthritis of the Hand, Hip, and Knee. Arthritis Care Res (Hoboken) 72, 149162, doi:10.1002/acr.24131 (2020). 
Arden, N. K. et al. Non-surgical management of knee osteoarthritis: comparison of ESCEO and OARSI 2019 guidelines. Nat Rev Rheumatol 17, 59-66, doi:10.1038/s41584-020-00523-9 (2021).

7 Geenen, R. et al. EULAR recommendations for the health professional's approach to pain management in inflammatory arthritis and osteoarthritis. Ann Rheum Dis 77, 797-807, doi:10.1136/annrheumdis-2017-212662 (2018).

8 Filardo, G. et al. Platelet-rich plasma intra-articular knee injections for the treatment of degenerative cartilage lesions and osteoarthritis. Knee Surg Sports Traumatol Arthrosc 19, 528-535, doi:10.1007/s00167-010-1238-6 (2011).

9 Gobbi, A., Karnatzikos, G., Mahajan, V. \& Malchira, S. Platelet-rich plasma treatment in symptomatic patients with knee osteoarthritis: preliminary results in a group of active patients. Sports Health 4, 162-172, doi:10.1177/1941738111431801 (2012).

10 De La Mata, J. Platelet rich plasma. A new treatment tool for the rheumatologist? Reumatol Clin 9, 166-171, doi:10.1016/j.reuma.2012.05.011 (2013).

11 Jang, S. J., Kim, J. D. \& Cha, S. S. Platelet-rich plasma (PRP) injections as an effective treatment for early osteoarthritis. Eur J Orthop Surg Traumato/ 23, 573-580, doi:10.1007/s00590-012-1037-5 (2013).

12 Dold, A. P., Zywiel, M. G., Taylor, D. W., Dwyer, T. \& Theodoropoulos, J. Platelet-rich plasma in the management of articular cartilage pathology: a systematic review. Clin J Sport Med 24, 31-43, doi:10.1097/01.jsm.0000432855.85143.e5 (2014).

13 Filardo, G. et al. Leukocyte-poor PRP application for the treatment of knee osteoarthritis. Joints 1, 112-120 (2013).

14 Sundman, E. A. et al. The anti-inflammatory and matrix restorative mechanisms of platelet-rich plasma in osteoarthritis. Am J Sports Med 42, 35-41, doi:10.1177/0363546513507766 (2014).

15 Pavone, V. et al. Injection-Based Management of Osteoarthritis of the Knee: A Systematic Review of Guidelines. Front Pharmacol 12, 661805, doi:10.3389/fphar.2021.661805 (2021).

16 van Buul, G. M. et al. Platelet-rich plasma releasate inhibits inflammatory processes in osteoarthritic chondrocytes. Am J Sports Med 39, 2362-2370, doi:10.1177/0363546511419278 (2011).

17 Gato-Calvo, L., Hermida-Gomez, T., Romero, C. R., Burguera, E. F. \& Blanco, F. J. Anti-Inflammatory Effects of Novel Standardized Platelet Rich Plasma Releasates on Knee Osteoarthritic Chondrocytes and Cartilage in vitro. Curr Pharm Biotechnol 20, 920-933, doi:10.2174/1389201020666190619111118 (2019).

18 Chou, S. H. \& Shih, C. L. Efficacy of different platelet-rich plasma injections in the treatment of mild-moderate knee osteoarthritis: A systematic review and meta-analysis. Int J Clin Pract 75, e14068, 
doi:10.1111/ijcp.14068 (2021).

19 Nie, L. Y., Zhao, K., Ruan, J. \& Xue, J. Effectiveness of Platelet-Rich Plasma in the Treatment of Knee Osteoarthritis: A Meta-analysis of Randomized Controlled Clinical Trials. Orthop J Sports Med $\mathbf{9}$, 2325967120973284, doi:10.1177/2325967120973284 (2021).

20 Aiyer, R. et al. Treatment of knee osteoarthritic pain with platelet-rich plasma: a systematic review of clinical studies. Pain Manag 11, 419-431, doi:10.2217/pmt-2020-0052 (2021).

21 Eymard, F. et al. Intra-articular injections of platelet-rich plasma in symptomatic knee osteoarthritis: a consensus statement from French-speaking experts. Knee Surg Sports Traumatol Arthrosc, doi:10.1007/s00167-020-06102-5 (2020).

22 Kellgren, J. H. \& Lawrence, J. S. Radiological assessment of osteo-arthrosis. Ann Rheum Dis 16, 494-502, doi:10.1136/ard.16.4.494 (1957).

23 Kaneko, S. et al. Interleukin-6 and interleukin-8 levels in serum and synovial fluid of patients with osteoarthritis. Cytokines Cell Mol Ther 6, 71-79, doi:10.1080/13684730050515796 (2000).

24 Mathiessen, A. \& Conaghan, P. G. Synovitis in osteoarthritis: current understanding with therapeutic implications. Arthritis Res Ther 19, 18, doi:10.1186/s13075-017-1229-9 (2017).

25 Bai, Y. et al. [Change of inflammatory cytokines levels in both synovial fluid and plasm of patients with primary knee medical osteoarthritis after high tibial osteotomy]. Zhongguo Xiu Fu Chong Jian Wai Ke Za Zhi 31, 422-426, doi:10.7507/1002-1892.201609123 (2017).

26 Shimura, Y. et al. Serum interleukin 6 levels are associated with depressive state of the patients with knee osteoarthritis irrespective of disease severity. Clin Rheumato/ 36, 2781-2787, doi:10.1007/s10067-017-3826-z (2017).

27 Filardo, G. et al. Platelet-rich plasma intra-articular injections for cartilage degeneration and osteoarthritis: single- versus double-spinning approach. Knee Surg Sports Traumatol Arthrosc 20, 20822091, doi:10.1007/s00167-011-1837-x (2012).

28 Joshi Jubert, N., Rodriguez, L., Reverte-Vinaixa, M. M. \& Navarro, A. Platelet-Rich Plasma Injections for Advanced Knee Osteoarthritis: A Prospective, Randomized, Double-Blinded Clinical Trial. Orthop J Sports Med 5, 2325967116689386, doi:10.1177/2325967116689386 (2017).

29 Park, Y. B., Kim, J. H., Ha, C. W. \& Lee, D. H. Clinical Efficacy of Platelet-Rich Plasma Injection and Its Association With Growth Factors in the Treatment of Mild to Moderate Knee Osteoarthritis: A Randomized Double-Blind Controlled Clinical Trial As Compared With Hyaluronic Acid. Am J Sports Med 49, 487-496, doi:10.1177/0363546520986867 (2021). 
Randomized Controlled Trials. Cartilage, 1947603520931170, doi:10.1177/1947603520931170 (2020).

31 Manner, P. A., Tubb, C. C. \& Levine, B. R. AAOS Appropriate Use Criteria: Surgical Management of Osteoarthritis of the Knee. J Am Acad Orthop Surg 26, e194-e197, doi:10.5435/JAAOS-D-17-00425 (2018).

\section{Tables}

Due to technical limitations, table 1 is only available as a download in the Supplemental Files section.

\section{Figures}

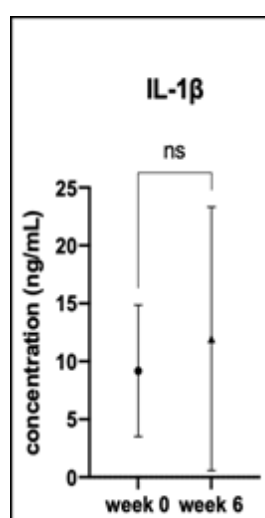

IL-17A
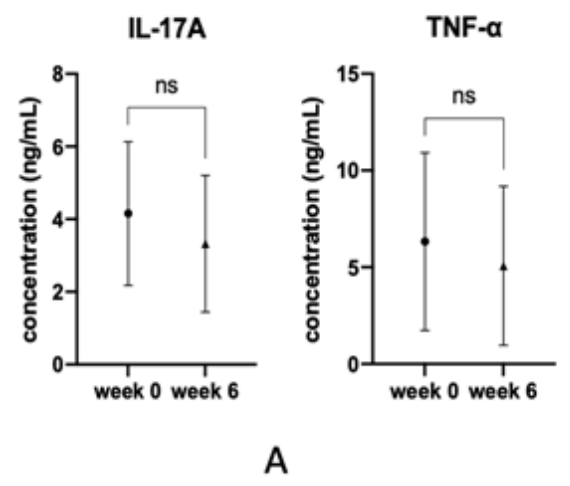

IL-6

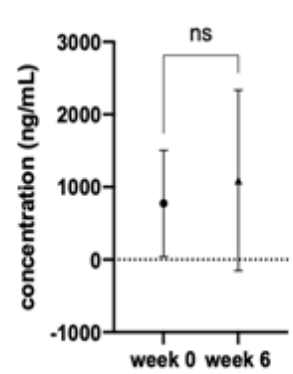

A

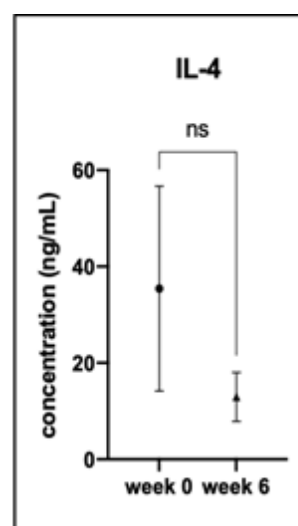

IL-13

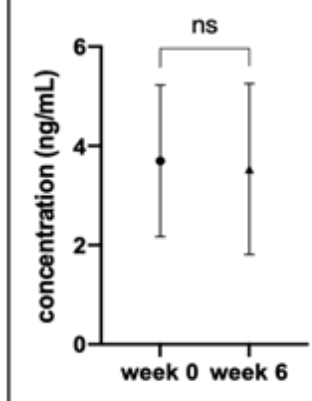

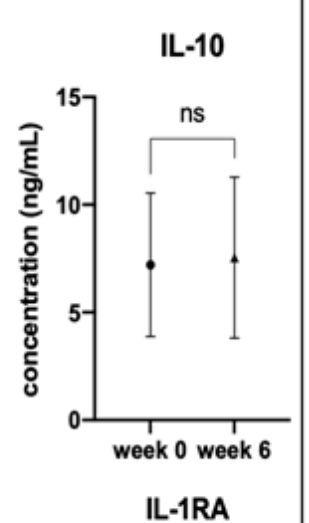

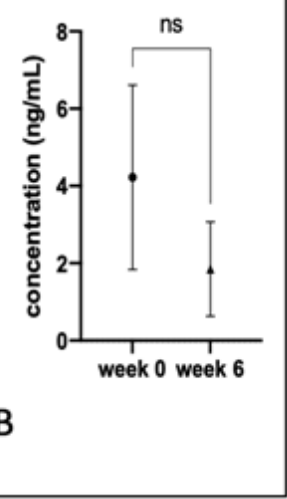

\section{Figure 1}

Demonstrating no differences in levels of synovial cytokines and growth factors of group A at 6 weeks of FU, which the baseline levels were performed at week 0 before the first PRP injection. A: Inflammatory cytokines including IL-1ß, IL-6, IA-17A, and TNF-alpha B: Anti-inflammatory cytokines including IL-4, IL-10, IL-13, and IL-1RA C: Growth factors including TGF-B1, VEGF, PDGF-AA, and PDGF-BB 

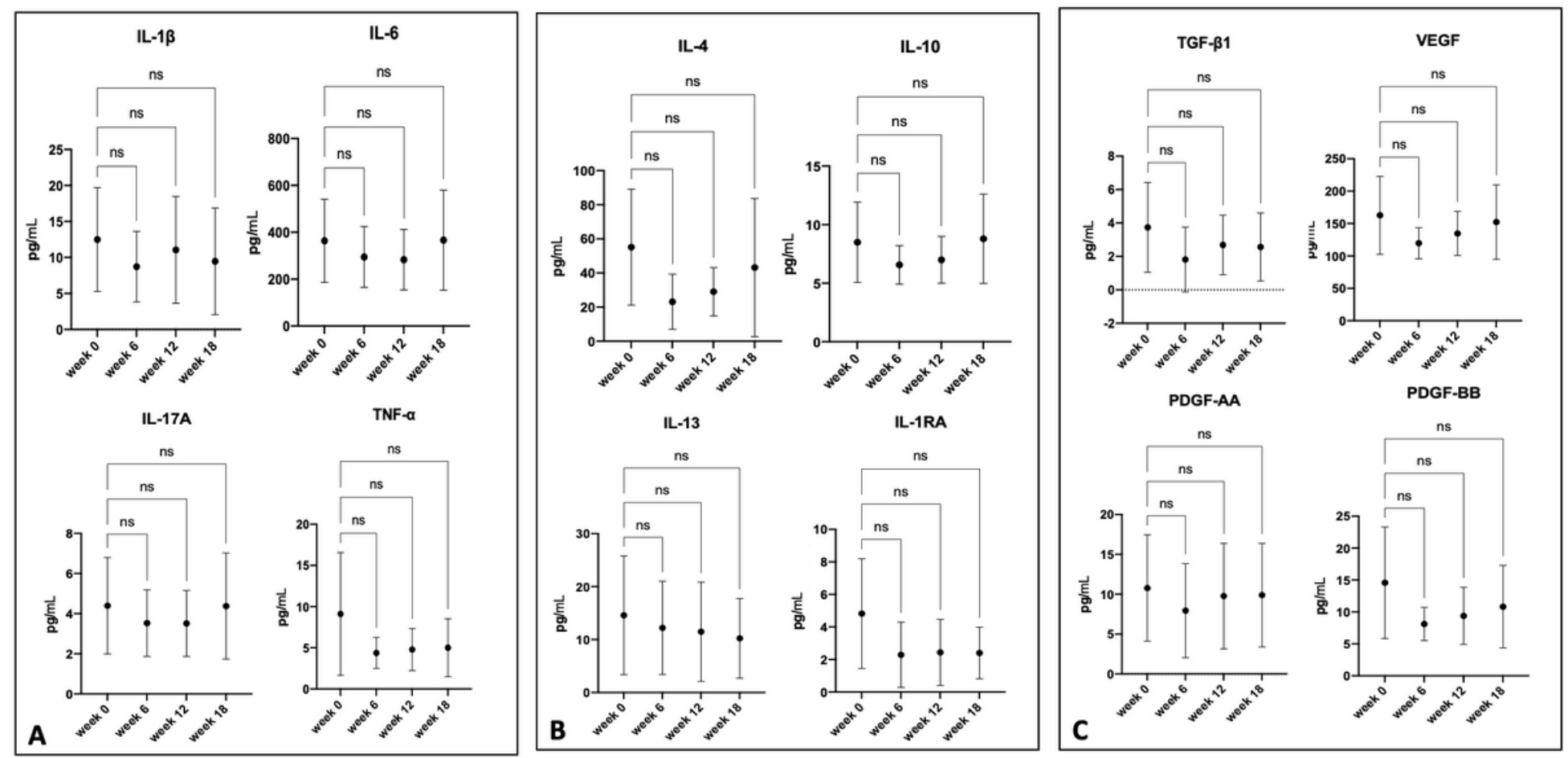

Figure 2

Demonstrating no differences in levels of synovial cytokines and growth factors of group B at 6 weeks, 12 weeks, and 18 weeks of $\mathrm{FU}$, which the baseline levels were performed at week 0 before the first PRP injection. A: Inflammatory cytokines including IL-1ß, IL-6, IA-17A, and TNF-alpha B: Anti-inflammatory cytokines including IL-4, IL-10, IL-13, and IL-1RA C: Growth factors including TGF-B1, VEGF, PDGF-AA, and PDGF-BB

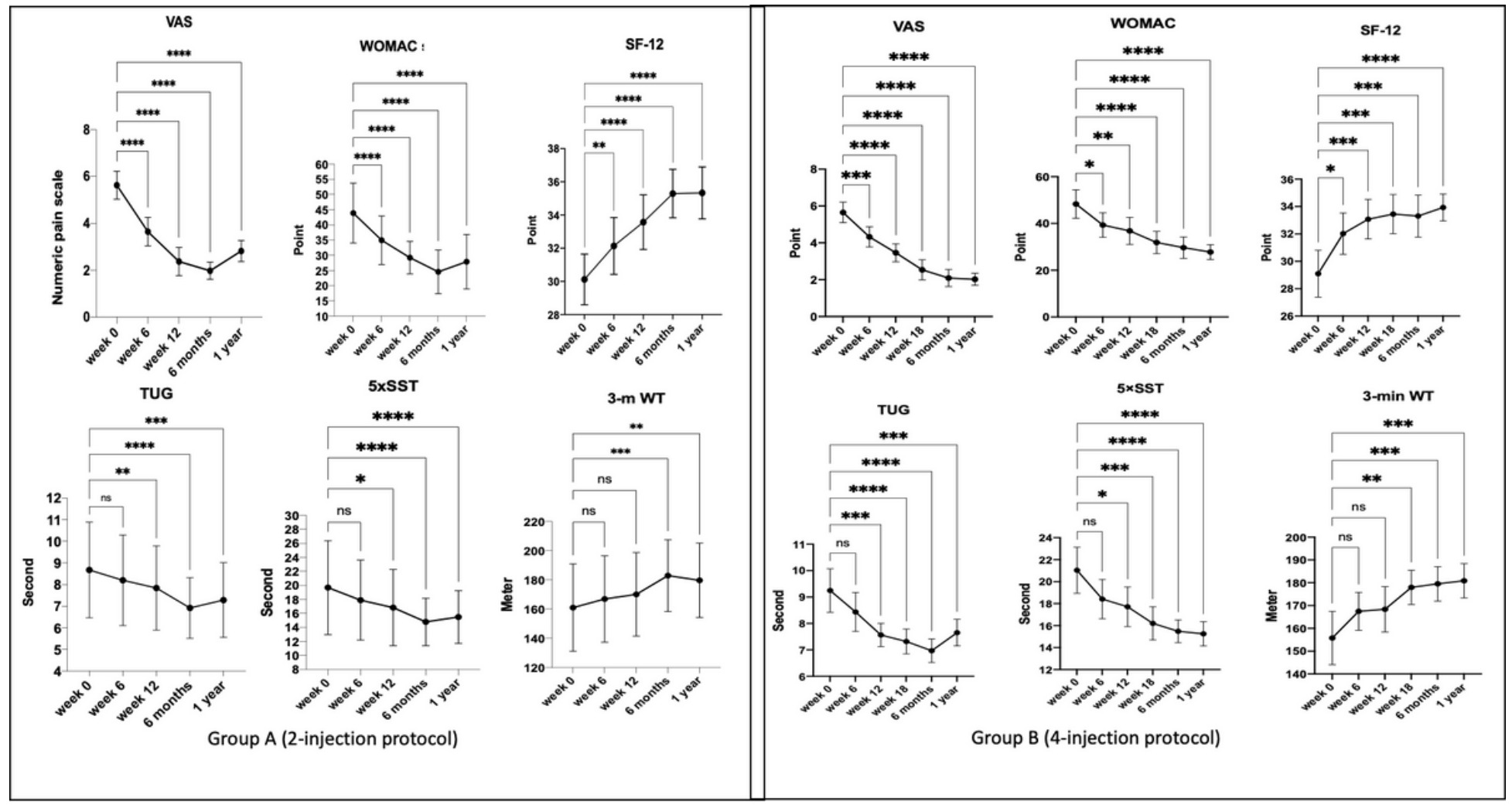


Figure 3

Demonstrating similarly changes of clinical outcomes of both group A and B from baseline (week 0 ) to 6 weeks, 12 weeks, 18 weeks (only in group B), 6 months, and one year of FU. The VAS, WOMAC index and SF-12 significantly improved from 6 weeks to one year in both groups. The TUG and $5 \times$ SST significantly improved from 12 weeks to one year in both groups. The 3-m WT significantly improved from 6 months and 18 weeks to one year in group $A$ and $B$, respectively.
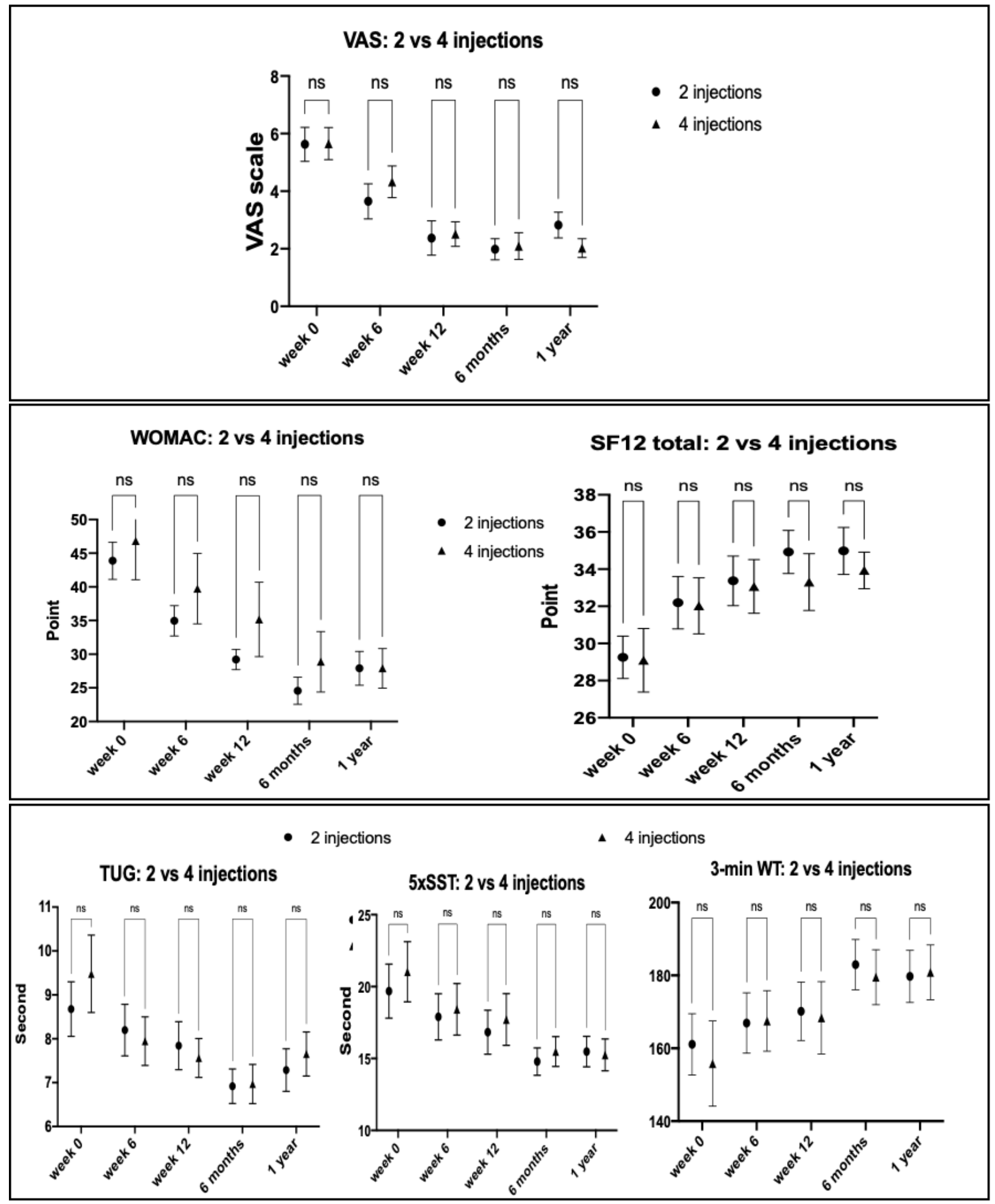
Figure 4

Comparing changes of clinical outcomes, including VAS, WOMAC, SF-12, TUG, $5 \times$ SST, and 3-m WT between group $A$ and $B$ from the baseline (week 0 ) to 6 weeks, 12 weeks, six months, and one year after PRP injections with no significant differences at all FU visits.

\section{Supplementary Files}

This is a list of supplementary files associated with this preprint. Click to download.

- Table1.png 